\title{
COMPORTAMENTO DA COR DE LÂMINAS DE MADEIRA DE PAU-MARFIM (Balfourodendron riedelianum) TRATADA COM PRODUTOS DE ACABAMENTO1
}

\author{
Clarissa Melo Lima², Joaquim Carlos Gonçalez ${ }^{4}$, Tito Ricardo Vaz da Costa ${ }^{3}$, Reginaldo Sergio Pereira ${ }^{4}$,
} João Batista Melo Lima ${ }^{5}$ e Maria do Socorro Alves Lima 6

\begin{abstract}
RESUMO - Este trabalho testou produtos no combate ao envelhecimento da madeira. Através de uma câmara de envelhecimento e aparelho de espectrocolorimetria, caracterizou-se a influência de produtos químicos de acabamentos aplicados à madeira durante processo artificial de fotodegradação. Acompanhou-se o envelhecimento do Pau-marfim (Balfourodendron riedelianum) por 8.807 medições colorimétricas, após receber ciclos de radiação ultravioleta durante períodos: $20,40,60,80,120,180,250,310,410$ e 500 horas. O sistema CIE La*b* de 1976 mostrou as modificações das cores. O uso da seladora provocou uma maior variação dos parâmetros colorimétricos da madeira quando comparado aos demais tratamentos.
\end{abstract}

Palavras-chave: Envelhecimento, Radiação, Colorimetria.

\section{COLOR BEHAVIOR OF PAU-MARFIM (Balfourodendron riedelianum) WOOD LAMINATES TREATED WITH FINISHING PRODUCTS.}

\begin{abstract}
This study tested products to combat aging of wood. Through an aging chamber and a spectrocolorimetry device, the influence of chemical finishing products applied to wood were characterized during artificial photodegradation process. The aging of Balfourodendron riedelianum was monitored through 8.807 colorimetry measurements, with the samples undergoing cycles of ultraviolet radiation for periods of: 20, 40, 60, 80, 120, 180, 250, 310, 410 and 500 hours. The data was loaded to Excel and processed. The CIE La*b* system of 1976 showed the color changes. The use of a sealant caused a greater variation in the wood's colorimetric parameters when compared to other treatment methods.
\end{abstract}

Keywords: Aging, Radiation, Colorimetry.

\section{INTRODUÇÃ̃O}

A madeira de pau-marfim (Balfourodendron riedelianum) possui densidade aparente a $15 \%$ de umidade entre 0,80 e 0,90 g. $\mathrm{cm}^{-3}$ e densidade básica entre 0,69 e $0,73 \mathrm{~g} \cdot \mathrm{cm}^{-3}$. É considerada madeira densa (JANKOWSKY et al., 1990).

A espécie pode ser usada para fabricação de móveis de luxo, molduras, guarnições internas, portas, artefatos domésticos, peças torneadas, laminados decorativos, tacos para assoalhos, carpintaria e marcenaria em geral (LORENZI, 1992). No Brasil, a lâmina de madeira de pau-marfim é muito valorizada devido à dificuldade de ser encontrada (ANGELI et al., 2005).

O efeito das intempéries provoca degradação física na madeira, mudanças de cor, reações químicas de seus contribuintes, aspereza superficial, rachaduras e fissuras.

\footnotetext{
${ }^{1}$ Recebido em 29.03.2012 aceito para publicação em 09.04.2013.

${ }^{2}$ Programa de Pós- Graduação em Ciência Florestal, Departamento de Engenharia Florestal, Universidade de Brasília. E-mail:<clarissaufc@gmail.com>.

${ }_{3}^{3}$ Programa de Pós-Graduação em Engenharia Florestal na Universidade de Brasília. E-mail: < goncalez@unb.br>e < reginaldosp@unb.br>.

${ }^{4}$ Programa de Pós-Graduação em Engenharia Elétrica na Universidade de Brasília. E-mail: <titovaz@ gmail.com>.

${ }^{5}$ Departamento de Geotecnia, Universidade Federal da Paraíba. E mail:<jbmlima@yahoo.com.br>.

${ }^{6}$ Universidade Federal do Ceará, UFC. E-mail: <socorrinhaalves@ yahoo.com.br>.
} 
Na deterioração química também se verifica a mudança na coloração da madeira (PAULILO et al., 2010).

O fenômeno da fotodegradação da madeira é superficial. Isso ocorre em função da variedade de sistemas (quinomas, bifenil etc.) e dos grupos cromóforos (carbonila, dupla ligação etc.) da sua camada externa. Essas formações impedem a entrada de luz na madeira.

Segundo Hon (2001), a radiação UV pode penetrar a uma profundidade de até $75 \mu \mathrm{m}$, enquanto a luz visível, até $200 \mu \mathrm{m}$. Contudo, embora a luz visível tenha maior capacidade de penetração, a energia é menor que $70 \mathrm{kcal} / \mathrm{mol}$, o que é insuficiente para romper as ligações químicas dos elementos constituintes da madeira (PASTORE, 2004).

As variações na coloração da madeira estão associadas, principalmente, com a formação e fotodecomposição de produtos cromóforos da lignina (PASTORE, 2004).

A modificação da cor das madeiras pode ser mensurada pelo sistema CIE-L*a*b*. Esse sistema foi desenvolvido pela Comission International d'Eclairage - CIE e tem por objetivos controlar a qualidade e permitir a reprodução da coloração de produtos industrializados. Por esse método, a percepção física das cores se dá em um espaço tridimensional, de acordo com as variações da claridade ou luminosidade $\Delta \mathrm{L}^{*}$ e as coordenadas cromáticas $\Delta \mathrm{a}^{*} \mathrm{e} \Delta \mathrm{b} *$, que correspondem aos pares de cores vermelho-verde e amarelo-azul, respectivamente (HON, 2001). Os pares de cores são representados por números que vão de $-120 \mathrm{a}+120$. No caso da coordenada " $a$ *, o valor -120 representa a cor verde e o valor +120 , a cor vermelha. Para a coordenada " $b$ *, o valor -120 representa a cor azul e o valor +120 , a cor amarela.

Esta pesquisa teve por objetivo verificar o comportamento da cor de lâminas de madeira de paumarfim (B. riedelianum) tratada com dois produtos de acabamento (óleo de peroba e seladora) colocados na presença de radiação ultravioleta (UV) artificial.

\section{MATERIAL E MÉTODOS}

Este trabalho realizou-se no Laboratório de Tecnologia da Madeira do Departamento de Engenharia Florestal da Universidade de Brasília.

As lâminas de madeira da espécie foram adquiridas no mercado brasiliense e apresentavam comprimento inicial de $250 \mathrm{~cm}, 14 \mathrm{~cm}$ de largura e aproximadamente $1 \mathrm{~mm}$ de espessura.

A partir dessas lâminas foram confeccionados 32 corpos de provas, com dimensões de $1,0 \mathrm{~mm}$ x 7,0 cm x 5,0 cm (espessura x comprimento x largura). Em seguida, os corpos de prova foram lixados com lixa grana 100 . Posteriormente, essas amostras foram submetidas a quatro tratamentos distintos: tratamento 1: oito corpos de provas com as lâminas de madeira em estado natural (testemunha); tratamento 2: oito corpos de prova tratados com o produto de acabamento óleo de peroba; tratamento 3: oito corpos de prova tratados com o produto de acabamento seladora; e tratamento 4: oito corpos de prova tratados com uma combinação de seladora e óleo de peroba, conforme procedimentos usados na indústria, isto é, os oito corpos de prova foram tratados inicialmente com seladora, para fazer o fundo; e, para finalizar, utilizouse óleo de peroba.

O óleo de peroba é um produto químico feito com base em extratos vegetais e composto dos seguintes ingredientes: óleo mineral, óleo vegetal, solvente mineral, solvente vegetal e aromatizante.

A seladora é um produto químico sintético composto dos seguintes ingredientes: resina maleica, hidrocarbonetos aromáticos (exceto benzeno), cetonas, minerais inertes, alcoóis, ésteres e algodão colódio. Essa seladora foi classificada conforme a norma NBR 11702/1992, tipo 4.1.10, ABNT (2010).

Após a secagem da primeira camada, um novo lixamento manual foi realizado com lixa grana 150 . Em seguida, os corpos de provas receberam uma segunda camada dos produtos correspondentes a cada tratamento. Por fim, repetiu-se o lixamento manual com lixa grana 220, e aplicou-se a terceira demão dos produtos já citados. Os procedimentos de acabamentos $\left(1^{\mathrm{a}}, 2^{\mathrm{a}}\right.$ e $3^{\text {a }}$ demãos de produtos) são os utilizados normalmente pelos industriais de mobiliário no Brasil.

Concluído o processo de acabamento, as amostras seguiram para medição da cor no espectrofotômetro. Para acompanhamento do processo de fotodegradação foram registrados os valores dos parâmetros colorimétricos $\mathrm{L}^{*}$ (luminosidade ou claridade), a* (posição da cor sobre o eixo verde-vermelho), b* (posição da cor sobre o eixo azul-amarelo), C (cromaticidade ou saturação) e h* (ângulo de tinta), de acordo com o Sistema CIELab 1976. O espectrofotômetro utilizado

Revista Árvore, Viçosa-MG, v.37, n.2, p.377-384, 2013 
no trabalho foi o Color Eye XTH - X-Rite, com iluminante D65 e ângulo de $10^{\circ} \mathrm{C}$ acoplado a um computador com o software Color iControl. Os parâmetros colorimétricos de todas as amostras foram medidos antes das aplicações dos produtos de acabamento, após a aplicação dos produtos e a cada intervalo de tempo dos tratamentos na câmara de envelhecimento acelerado da Q-Lab, modelo QUV/spray.

Os ciclos de radiação UV utilizados nesta pesquisa foram 20, 40, 60, 80, 120, 180, 250, 310, 410 e $500 \mathrm{~h}$. As amostras de madeiras foram colocadas dentro da câmara de envelhecimento (QUV) para receber ciclos de radiação UV durante o período inicial de 20 h. Em seguida, as amostras foram retiradas da câmara, e tomou-se a medida da cor. As amostras retornaram à câmara de envelhecimento para receber mais $20 \mathrm{~h}$ de radiação UV. Esse procedimento foi feito para todos os tempos mencionados até se completar o período de $500 \mathrm{~h}$. Cada corpo de prova recebeu 25 leituras, fazendo-se uma varredura em toda a superfície da amostra (face tangencial).

Após as medições, foi calculada a média de cada um dos parâmetros colorimétricos em cada medição, com o propósito de verificar a existência de variação ao longo dos intervalos de exposição aos raios UVs, através da análise de variância (ANOVA) e do teste de médias de Tukey a 5\% de significância. A comparação estatística das médias seguiu a mesma metodologia utilizada por Souza et al. (2012). Para a mensuração da alteração de cor nas amostras, combinando as variáveis $\mathrm{L}^{*}, \mathrm{a}^{*} \mathrm{e} \mathrm{b}^{*}$, antes e depois das exposições $(\Delta \mathrm{E})$, foi utilizada a seguinte fórmula (equação 1):

$$
\Delta \mathrm{E}=\sqrt{\Delta \mathrm{L}^{2}+\Delta \mathrm{a}^{2}+\Delta \mathrm{b}^{2}}
$$

Para verificar a variação total da cor $(\Delta \mathrm{E})$ em cada tratamento, utilizou-se a Tabela 1, proposta por Hikita

Tabela 1 - Classificação da variação total da cor $(\Delta \mathrm{E})$ de madeiras.

Table 1 -Classification of the total color variation $(\Delta E)$ the of wood.

\begin{tabular}{cc}
\hline Variação da cor $(\Delta \mathrm{E})$ & Classificação \\
\hline $0,0-0,5$ & Desprezível \\
$0,5-1,5$ & Ligeiramente perceptível \\
$1,5-3,0$ & Notável \\
$3,0-6,0$ & Apreciável \\
$6,0-12,0$ & Muito apreciável \\
\hline
\end{tabular}

et al. (2001). Essa tabela está baseada em níveis de percepção, e faz uma classificação da variação total da $\operatorname{cor}(\Delta \mathrm{E})$ de madeiras.

\section{RESULTADOS}

Os valores médios dos parâmetros colorimétricos dos quatro tratamentos durante os ciclos de exposição UV das amostras de lâminas de pau-marfim são apresentados na Tabela 2 .

Os valores médios referentes a um mesmo ciclo de tempo diferem significativamente entre si, considerando-se um intervalo de confiança de $5 \%$ para o teste de comparação de médias de Tukey.

As variações dos valores dos parâmetros colorimétricos provocadas pelos 11 ciclos de radiação $\mathrm{UV}$, num total de $500 \mathrm{~h}$ de radiação, encontram-se na Tabela 3 .

\section{DISCUSSÃO}

Em todos os tratamentos, as coordenadas a*e $\mathrm{b} *$ exibiram valores positivos. Isso significa que as amostras apresentaram cromaticidade vermelha e amarela. Com base em Camargos e Gonçalez (2001), a luminosidade é considerada escura para o valor de $\mathrm{L}^{*}$ menor ou igual a $56 \mathrm{e}$, como cor clara, para o valor de L* acima de 56. Seguindo essa classificação, pode-se afirmar que as amostras pertencentes ao tratamento 1 permaneceram claras durante todo o ciclo de radiação. As amostras dos demais tratamentos eram inicialmente claras e foram escurecendo à medida que eram submetidas à radiação UV.

Relativo à formação da cor, pôde-se observar tendência de acentuação da coloração vermelha em todos os tratamentos, uma vez que os valores da coordenada a* (gama de cor vermelha) cresceram em proporção direta às horas de radiação UV. Observou-se, ainda, tendência geral de aumento gradual dos valores da coordenada b*, matiz amarela, até o ciclo de medição de 310 h, seguido de diminuição desse indicador. O comportamento indica a existência de um ponto de inflexão da curva de b*, com diminuição da componente amarela a partir desse período de incidência luminosa.

No tratamento 3 , a coordenada $a^{*}$ atingiu seu maior valor $(21,26)$, após 500 h de incidência de radiação UV, resultando na classificação da cor mais fosca da lamina de madeira de pau-marfim. Os resultados indicaram 
Tabela 2 - Valores médios dos parâmetros colorimétricos de laminas de madeira Balfourodendron riedelianum para os quatro tratamentos e os tempos de radiação estudados.

Table 2 - Mean values of colorimetric parameters for Balfourodendron riedelianum wood blades for the four treatments and irradiation times studied.

\begin{tabular}{|c|c|c|c|c|c|c|c|}
\hline Tratamento & Radiação UV (horas) & $\mathrm{L}^{*}$ & $a^{*}$ & $\mathrm{~b}^{*}$ & $\mathrm{C}$ & $\mathrm{h}^{*}$ & Nome da cor $(* *)$ \\
\hline \multirow{11}{*}{1 (Testemunho) } & 0 & $70,32 \mathrm{~A}$ & $6,98 \mathrm{~A}$ & $28,49 \mathrm{~A}$ & $29,33 \mathrm{~A}$ & $76,24 \mathrm{~A}$ & \multirow[t]{10}{*}{ Amarelo Claro } \\
\hline & 20 & $70,66 \mathrm{~B}$ & $7,05 \mathrm{~A}$ & $28,60 \mathrm{~B}$ & $29,46 \mathrm{~A}$ & $76,16 \mathrm{~A}$ & \\
\hline & 40 & $68,37 \mathrm{C}$ & $9,64 \mathrm{~B}$ & $36,49 \mathrm{C}$ & $37,74 \mathrm{~B}$ & $75,20 \mathrm{~B}$ & \\
\hline & 60 & $65,13 \mathrm{D}$ & $11,14 \mathrm{C}$ & $38,27 \mathrm{D}$ & $39,86 \mathrm{C}$ & $73,77 \mathrm{C}$ & \\
\hline & 80 & $64,89 \mathrm{~F}$ & $11,53 \mathrm{D}$ & $38,47 \mathrm{C}$ & $40,16 \mathrm{D}$ & $73,32 \mathrm{D}$ & \\
\hline & 120 & $63,56 \mathrm{G}$ & $11,80 \mathrm{E}$ & $38,14 \mathrm{D}$ & $39,92 \mathrm{C}$ & $72,81 \mathrm{E}$ & \\
\hline & 180 & $64,08 \mathrm{H}$ & $12,60 \mathrm{~F}$ & $38,90 \mathrm{E}$ & $40,89 \mathrm{E}$ & $72,05 \mathrm{~F}$ & \\
\hline & 250 & $63,56 \mathrm{G}$ & $12,96 \mathrm{G}$ & $38,51 \mathrm{C}$ & $40,64 \mathrm{~F}$ & $71,40 \mathrm{G}$ & \\
\hline & 310 & $63,64 \mathrm{G}$ & $13,83 \mathrm{H}$ & $39,26 \mathrm{~F}$ & $41,62 \mathrm{G}$ & $70,60 \mathrm{H}$ & \\
\hline & 410 & $62,06 \mathrm{I}$ & $13,87 \mathrm{H}$ & $38,41 \mathrm{C}$ & $40,84 \mathrm{E}$ & $70,14 \mathrm{I}$ & \\
\hline & 500 & $61,49 \mathrm{I}$ & $13,70 \mathrm{I}$ & $37,10 \mathrm{G}$ & $39,55 \mathrm{H}$ & $69,73 \mathrm{~J}$ & Amarelo Alaranjado \\
\hline \multirow{11}{*}{2 (Óleo de peroba) } & 0 & $70,32 \mathrm{~A}$ & $6,98 \mathrm{~A}$ & $28,49 \mathrm{~A}$ & $29,33 \mathrm{~A}$ & $76,24 \mathrm{~A}$ & \multirow[t]{10}{*}{ Amarelo Claro } \\
\hline & 20 & $56,62 \mathrm{~B}$ & $12,63 \mathrm{~B}$ & $35,53 \mathrm{~B}$ & $37,72 \mathrm{~B}$ & $70,43 \mathrm{~B}$ & \\
\hline & 40 & $55,72 \mathrm{C}$ & $14,10 \mathrm{C}$ & $36,87 \mathrm{C}$ & $39,49 \mathrm{C}$ & $69,06 \mathrm{C}$ & \\
\hline & 60 & $53,34 \mathrm{H}$ & $14,43 \mathrm{D}$ & $36,79 \mathrm{D}$ & $39,53 \mathrm{C}$ & $68,57 \mathrm{D}$ & \\
\hline & 80 & $53,73 \mathrm{H}$ & $14,48 \mathrm{D}$ & $37,29 \mathrm{E}$ & $40,01 \mathrm{D}$ & $68,77 \mathrm{E}$ & \\
\hline & 120 & $53,69 \mathrm{H}$ & $14,56 \mathrm{E}$ & $37,45 \mathrm{~F}$ & $40,18 \mathrm{E}$ & $68,73 \mathrm{E}$ & \\
\hline & 180 & $54,50 \mathrm{I}$ & $15,16 \mathrm{H}$ & $38,65 \mathrm{G}$ & $41,52 \mathrm{~F}$ & $68,56 \mathrm{D}$ & \\
\hline & 250 & $54,56 \mathrm{I}$ & $15,47 \mathrm{I}$ & $39,46 \mathrm{H}$ & $42,39 \mathrm{G}$ & $68,58 \mathrm{D}$ & \\
\hline & 310 & $54,63 \mathrm{I}$ & $16,11 \mathrm{H}$ & $40,78 \mathrm{I}$ & $43,85 \mathrm{H}$ & $68,43 \mathrm{~F}$ & \\
\hline & 410 & $53,61 \mathrm{H}$ & $15,91 \mathrm{~J}$ & $39,63 \mathrm{H}$ & $42,71 \mathrm{I}$ & $68,11 \mathrm{G}$ & \\
\hline & 500 & $53,59 \mathrm{H}$ & $16,19 \mathrm{H}$ & $39,23 \mathrm{~J}$ & $42,45 \mathrm{G}$ & $67,56 \mathrm{H}$ & Amarelo Alaranjado \\
\hline \multirow{11}{*}{3 (Seladora) } & 0 & $70,32 \mathrm{~A}$ & $6,98 \mathrm{~A}$ & $28,49 \mathrm{~A}$ & $29,33 \mathrm{~A}$ & $76,24 \mathrm{~A}$ & \multirow[t]{11}{*}{ Amarelo Claro } \\
\hline & 20 & $66,78 \mathrm{~B}$ & $9,65 \mathrm{~B}$ & $34,90 \mathrm{~B}$ & $36,21 \mathrm{~B}$ & $74,55 \mathrm{~B}$ & \\
\hline & 40 & $60,22 \mathrm{C}$ & $15,70 \mathrm{C}$ & $41,66 \mathrm{C}$ & $44,52 \mathrm{C}$ & $69,37 \mathrm{C}$ & \\
\hline & 60 & $51,20 \mathrm{H}$ & $17,26 \mathrm{D}$ & $38,43 \mathrm{D}$ & $42,16 \mathrm{D}$ & $65,79 \mathrm{D}$ & \\
\hline & 80 & $46,95 \mathrm{I}$ & $16,26 \mathrm{E}$ & $34,55 \mathrm{D}$ & $38,22 \mathrm{E}$ & $64,72 \mathrm{E}$ & \\
\hline & 120 & $45,98 \mathrm{I}$ & $17,35 \mathrm{~F}$ & $34,02 \mathrm{E}$ & $38,25 \mathrm{E}$ & $62,83 \mathrm{~F}$ & \\
\hline & 180 & $47,49 \mathrm{~L}$ & $19,87 \mathrm{G}$ & $36,25 \mathrm{~F}$ & $41,42 \mathrm{~F}$ & $61,11 \mathrm{G}$ & \\
\hline & 250 & $46,72 \mathrm{I}$ & $20,29 \mathrm{H}$ & $36,50 \mathrm{G}$ & $41,84 \mathrm{G}$ & $60,72 \mathrm{H}$ & \\
\hline & 310 & $47,27 \mathrm{M}$ & $21,58 \mathrm{I}$ & $38,50 \mathrm{D}$ & $44,24 \mathrm{H}$ & $60,47 \mathrm{I}$ & \\
\hline & 410 & $46,32 \mathrm{~N}$ & $21,68 \mathrm{~J}$ & $37,18 \mathrm{H}$ & $43,13 \mathrm{I}$ & $59,53 \mathrm{~J}$ & \\
\hline & 500 & $45,54 \mathrm{I}$ & $21,26 \mathrm{~L}$ & $35,51 \mathrm{I}$ & $41,48 \mathrm{~F}$ & $58,83 \mathrm{~L}$ & \\
\hline \multirow{11}{*}{$\begin{array}{l}4 \text { (Seladora e } \\
\text { Óleo de peroba) }\end{array}$} & 0 & $70,32 \mathrm{~A}$ & $6,98 \mathrm{~A}$ & $28,49 \mathrm{~A}$ & $29,33 \mathrm{~A}$ & $76,24 \mathrm{~A}$ & \multirow{11}{*}{ Amarelo Claro } \\
\hline & 20 & $59,48 \mathrm{~B}$ & $11,70 \mathrm{~B}$ & $34,19 \mathrm{~B}$ & $36,15 \mathrm{~B}$ & $71,11 \mathrm{~B}$ & \\
\hline & 40 & $52,35 \mathrm{C}$ & $15,37 \mathrm{C}$ & $34,39 \mathrm{C}$ & $37,68 \mathrm{C}$ & $65,90 \mathrm{C}$ & \\
\hline & 60 & $47,77 \mathrm{D}$ & $15,82 \mathrm{D}$ & $30,13 \mathrm{D}$ & $34,06 \mathrm{D}$ & $62,18 \mathrm{D}$ & \\
\hline & 80 & $46,12 \mathrm{E}$ & $15,22 \mathrm{E}$ & $28,47 \mathrm{E}$ & $32,31 \mathrm{E}$ & $61,73 \mathrm{E}$ & \\
\hline & 120 & $46,63 \mathrm{~F}$ & $15,89 \mathrm{D}$ & $27,93 \mathrm{~F}$ & $32,17 \mathrm{~F}$ & $60,20 \mathrm{~F}$ & \\
\hline & 180 & $47,54 \mathrm{C}$ & $16,99 \mathrm{~F}$ & $28,52 \mathrm{G}$ & $33,26 \mathrm{G}$ & $59,01 \mathrm{G}$ & \\
\hline & 250 & $46,81 \mathrm{~F}$ & $17,41 \mathrm{G}$ & $28,13 \mathrm{H}$ & $33,14 \mathrm{G}$ & $57,97 \mathrm{H}$ & \\
\hline & 310 & $46,72 \mathrm{~F}$ & $18,18 \mathrm{H}$ & $29,28 \mathrm{I}$ & $34,53 \mathrm{H}$ & $57,90 \mathrm{H}$ & \\
\hline & 410 & $45,89 \mathrm{I}$ & $17,74 \mathrm{I}$ & $27,82 \mathrm{~J}$ & $33,05 \mathrm{I}$ & $57,20 \mathrm{I}$ & \\
\hline & 500 & 45,99I & $17,73 \mathrm{I}$ & $27,06 \mathrm{~L}$ & $32,42 \mathrm{E}$ & $56,39 \mathrm{~J}$ & \\
\hline
\end{tabular}

(**) Representa a cor da madeira conforme classificação proposta por Camargos e Gonçalez (2001).

$\mathrm{O}+\mathrm{P} \circledast$ lâmina de madeira + produto de acabamento.

Revista Árvore, Viçosa-MG, v.37, n.2, p.377-384, 2013 
Tabela 3 - Variações dos valores dos parâmetros de cor na superfície de laminas de madeira Balfourodendron riedelianum submetidos a diferentes tratamentos

Table 3 - Variations of the color parameter values of the surface of Balfourodendron riedelianum veneers subjected to different treatments

\begin{tabular}{|c|c|c|c|c|c|c|c|c|c|c|c|}
\hline \multirow[t]{2}{*}{ Parâmetro } & \multirow[t]{2}{*}{ Tratamento } & \multicolumn{10}{|c|}{ Tempo de radiação UV (horas) } \\
\hline & & 20 & 40 & 60 & 80 & 120 & 180 & 250 & 310 & 410 & 500 \\
\hline \multirow{4}{*}{$\Delta \mathrm{L}^{*}$} & 1 & 0,33 & $-2,28$ & $-3,25$ & $-0,24$ & $-1,33$ & 0,52 & $-0,52$ & 0,08 & $-1,58$ & $-0,57$ \\
\hline & 2 & $-14,53$ & $-0,90$ & $-2,38$ & 0,39 & $-0,04$ & 0,81 & 0,06 & 0,07 & $-1,02$ & $-0,02$ \\
\hline & 3 & $-4,23$ & $-6,56$ & $-9,02$ & $-4,25$ & $-0,97$ & 1,51 & $-0,77$ & 0,56 & $-0,96$ & $-0,78$ \\
\hline & 4 & $-11,04$ & $-7,13$ & $-4,58$ & $-1,65$ & 0,51 & 0,91 & $-0,73$ & $-0,08$ & $-0,83$ & 0,10 \\
\hline \multirow{4}{*}{$\Delta \mathrm{a}^{*}$} & 1 & 0,07 & 2,59 & 1,50 & 0,39 & 0,27 & 0,80 & 0,36 & 0,87 & 0,05 & $-0,17$ \\
\hline & 2 & 5,43 & 1,47 & 0,33 & 0,05 & 0,09 & 0,60 & 0,30 & 0,65 & $-0,20$ & 0,28 \\
\hline & 3 & $-4,23$ & $-6,56$ & $-9,02$ & $-4,25$ & $-0,97$ & 1,51 & $-0,77$ & 0,56 & $-0,96$ & $-0,78$ \\
\hline & 4 & $-11,04$ & $-7,13$ & $-4,58$ & $-1,65$ & 0,51 & 0,91 & $-0,73$ & $-0,08$ & $-0,83$ & 0,10 \\
\hline \multirow{4}{*}{$\Delta \mathrm{b}^{*}$} & 1 & 0,11 & 7,89 & 1,77 & 0,21 & $-0,33$ & 0,76 & $-0,39$ & 0,74 & $-0,85$ & $-1,31$ \\
\hline & 2 & 6,27 & 1,34 & $-0,08$ & 0,50 & 0,15 & 1,20 & 0,82 & 1,32 & $-1,15$ & $-0,40$ \\
\hline & 3 & 6,22 & 6,76 & $-3,23$ & $-3,88$ & $-0,53$ & 2,22 & 0,25 & 2,00 & $-1,32$ & $-1,67$ \\
\hline & 4 & 5,21 & 0,20 & $-4,26$ & $-1,66$ & $-0,54$ & 0,59 & $-0,40$ & 1,15 & $-1,46$ & $-0,76$ \\
\hline \multirow{4}{*}{$\Delta \mathrm{E}^{*}$} & 1 & 0,50 & 4,20 & 2,52 & 0,63 & 1,25 & 1,14 & 0,82 & 1,09 & 1,48 & 1,27 \\
\hline & 2 & 6,54 & 1,68 & 1,79 & 0,74 & 0,32 & 1,35 & 0,91 & 1,29 & 1,34 & 0,62 \\
\hline & 3 & 4,21 & 5,09 & 5,57 & 3,71 & 1,29 & 2,12 & 1,08 & 1,67 & 1,53 & 1,59 \\
\hline & 4 & 6,47 & 4,67 & 3,92 & 2,01 & 0,93 & 1,26 & 1,07 & 1,10 & 1,52 & 0,84 \\
\hline
\end{tabular}

que esse foi o tratamento menos eficiente do ponto de vista da conservação da matiz vermelha da cor da madeira.

As constatações mencionadas são confirmadas quando se analisa o comportamento da tonalidade (ângulo de tinta, $\mathrm{h}^{*}$ ) das amostras. Como os tratamentos estudados remetem a coloração das lâminas de madeira ao primeiro quadrante do sistema CIELAB 1976, pode-se considerar a variação do ângulo da variável h* entre $0^{\circ}$ e $90^{\circ}$, o que significa variação da matiz vermelha para a amarela. Observou-se em todos os tratamentos que as lâminas de madeira de pau-marfim tiveram diminuição no ângulo de tinta (variável h*), indicando influência na tonalidade vermelha.

Analisando a Tabela 3, constata-se que a partir dos valores negativos de $\Delta \mathrm{L}^{*}$ (variação de luminosidade) houve escurecimento na superfície das lâminas de pau-marfim em todos os tratamentos, ao final dos 11 ciclos de radiação UV. Percebe-se ainda, nessa tabela, que esse escurecimento foi mais representativo nas primeiras $80 \mathrm{~h}$ de radiação UV, muito embora se tenha observado tendência de escurecimento em todo o período analisado. Além disso, deve-se considerar que a simples aplicação de um produto altera a cor original da madeira, mesmo sendo esse produto transparente. Essa alteração inicial é no sentido de escurecer a madeira, independentemente da substância.
Observa-se que, no caso dos tratamentos 3 e 4, após cerca de $180 \mathrm{~h}$ de radiação houve inversão da declividade do $\Delta \mathrm{L}^{*}$, sugerindo o final do processo de escurecimento da madeira e, possivelmente, a estabilização (Figura 1). Apesar de, após esse tempo de irradiação, ainda haver oscilação da claridade $(\Delta \mathrm{L} *)$, contudo em valor $98 \%$ inferior ao do período anterior. Esses resultados podem auxiliar as empresas fabricantes de soluções de acabamentos da madeira na utilização de filtros para radiação UV em seus produtos.

Resultados semelhantes para o comportamento da variação de claridade $\left(\Delta \mathrm{L}^{*}\right)$ foram encontrados nas madeiras de jatobá (Hymenaea courbari) e de tauari (Couratari oblongifolia) sob radiação UV por um período de 168 h (COSTA et al., 2011). Em estudos semelhantes, verificou-se ainda que esse processo de clareamento com madeiras avermelhadas tende a estabilizar-se à medida que aumenta o tempo de tratamento (SILVA; PASTORE, 2004; SILVA et al., 2007).

Para o parâmetro $\Delta \mathrm{a}^{*}$, o tratamento 3 provocou a maior variação $(-25,47)$ e o tratamento 1 , o menor $(+6,72)$. Considerando o parâmetro $\Delta \mathrm{b}^{*}$, a maior variação foi observada no tratamento $2(+9,97)$ e a menor, no tratamento $4(-1,92)$. Em todos os casos, pode-se afirmar existir maior estabilidade para o amarelo, já que essa coordenada

Revista Árvore, Viçosa-MG, v.37, n.2, p.377-384, 2013 

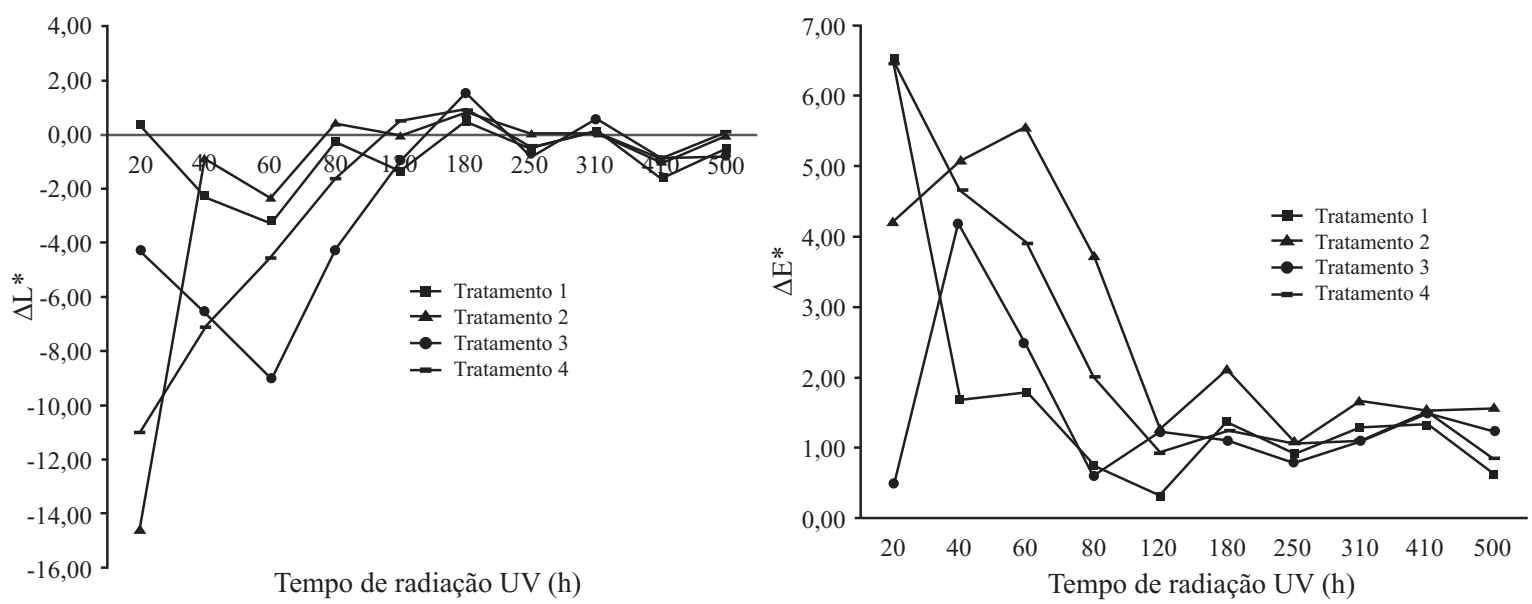

Figura 1 - Variação do parâmetro $\Delta \mathrm{L}^{*}$ e $\Delta \mathrm{E}^{*}$ provocada pela fotodegradação em lâminas de madeira de Balfourodendron riedelianum.

Figure 1 -Variations of $\Delta L^{*}$ and $\Delta E^{*}$ caused by photodegradation in veneers of Balfourodendron riedelianum.

cromática foi a que variou menos. Entre os produtos aplicados à madeira, a seladora foi a que causou maior instabilidade da cor durante os tempos estudados, uma característica indesejável. Entretanto, a madeira sem tratamento (testemunho) foi a que menos sofreu instabilidade da variação total de cor no decorrer dos tempos estudados.

A variação total da cor $\left(\Delta \mathrm{E}^{*}\right)$, determinada pela raiz quadrada da soma dos quadrados das variações de $\mathrm{L}^{*}, \mathrm{a}^{*}$ e b*, leva em consideração as diferenças das coordenadas cromáticas e de luminosidade, fornecendo uma visão geral sobre o desempenho das madeiras sob radiação UV.

Analisando ainda a Figura 1, pode-se afirmar que o desempenho do $\Delta \mathrm{E}^{*}$ nos quatro tratamentos teve comportamento semelhante a partir das $180 \mathrm{~h}$ de radiação, ou seja, apesar da alteração da cor na madeira de paumarfim, a variação total é ligeiramente perceptível $\left(0,5<\Delta \mathrm{E}^{*}<1,5\right)$. No entanto, no início das medições (até $180 \mathrm{~h}$ ), observou-se variação total de cor apreciável $\left(3<\Delta \mathrm{E}^{*}<6\right)$.

A refletância do pau-marfim em função do comprimento de onda pode ser observada na Figura 2. Nesse caso, apresentam-se curvas em intervalos de tempo de 0,120 e $500 \mathrm{~h}$ nos quatro tratamentos analisados.

Considerando o espectro de luz visível (400-700 $\mathrm{nm}$ ), foi observado um formato de onda-padrão do comprimento de onda versus reflectância do pau-marfim submetido aos quatro tratamentos distintos.
As menores variações de reflectância ao longo do tempo foram observadas no tratamento 1. Em todos os casos, o percentual dessa grandeza caiu entre os tempos 0 e $120 \mathrm{~h}$, seguido de aumento entre os tempos 120 e $500 \mathrm{~h}$.

\section{CONCLUSÕES}

A espectrocolorimetria possibilitou a obtenção de uma descrição objetiva da coloração das lâminas em estudo, quando submetidas à radiação UV artificial.

Os resultados da variação total da cor $\left(\Delta \mathrm{E}^{*}\right)$ sugerem que essa madeira apresenta maior resistência à radiação UV quando tratada com óleo de peroba ou quando mantida em seu estado natural.

Todos os tratamentos provocaram alterações nas cores da madeira com o passar do tempo. O uso da seladora acentuou a variação de cor. O tratamento com óleo de peroba preservou com melhor eficiência a cor original da madeira. A manutenção da cor original também ficou evidente nas amostras mantidas em seu estado natural.

A madeira mantida em seu estado original (tratamento 1) apresentou menor variação de refletância ao longo do período de exposição à radiação UV.

Considerando o efeito combinado da variação de cor e da variação de reflectância, conclui-se que a madeira de pau-marfim mantida em seu estado natural (tratamento 1) apresentou maior resistência à radiação UV. 

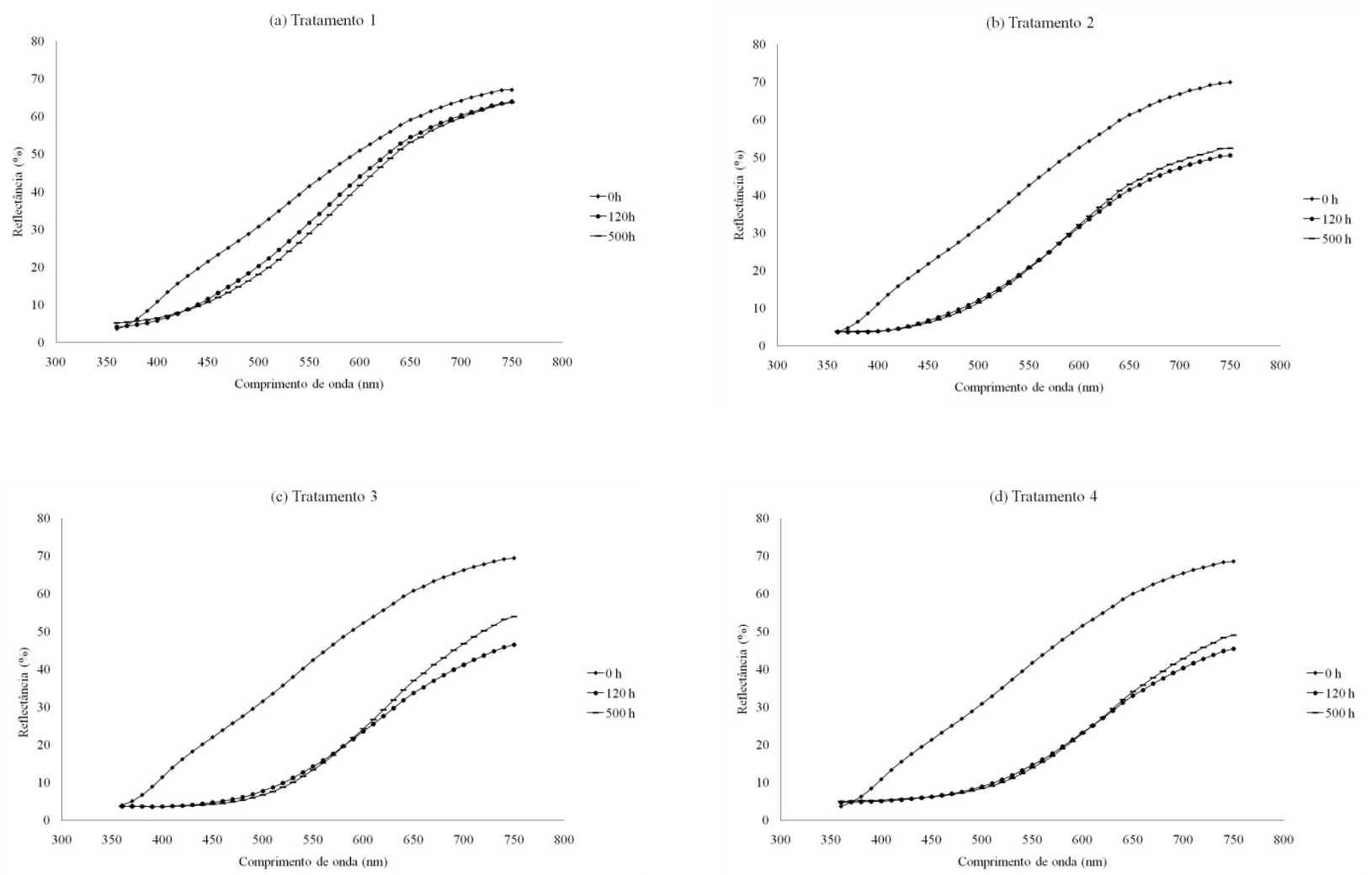

Figura 2 - Reflectância do Balfourodendron riedelianum submetido a diferentes tratamentos em função do comprimento de onda.

Figure 2 - Reflectance of Balfourodendron riedelianum subjected to different treatments as a function of wavelength.

\section{REFERÊNCIAS}

ANGELI, A.; BARRICHELO, L. E. G.; MÜLLER, P. $\mathrm{H}$. Balfourodendron riedelianum (Pau

Marfim). Piracicaba: Instituto de Pesquisa e Estudos Florestais - IPEF,. (Disponível em: http:// www.ipef.br/identificacao/

balfourodendron.riedelianum.asp), 2005.

ASSOCIAÇÃO BRASILEIRA DE NORMAS TÉCNICAS - ABNT. NBR 11702: Tintas para construção civil - Tintas para edificações não industriais -

Classificação. Rio de Janeiro: 2010.

CAMARGos, J. A. A.; GONÇALEZ, J. C. A colorimetria aplicada como instrumento na elaboração de uma tabela de cores de madeira. Brasília: MMA, 2001. (Brasil Florestal, 71).
COSTA, J. A. et al. Fotodegradação de duas espécies tropicais: JATOBÁ (Hymenaea courbaril) e TAUARI (Couratari oblongifolia) submetidas à radiação ultravioleta. Cerne, v.17, n.1, p.133-139, 2011.

HIKITA, Y.; TOYODA, T.; AZUMA, M.

Weathering testing of timber: discoloration. In:

IMAMURA, Y. High performance utilization of wood for outdooor uses. Kyoto: Press-Net, 2001.

HON, D. N. S. Weathering and photochemistry of wood. In: HON, D. N. S.; SHIRAISHI, N. Wood and cellulosic chemistry. 2.ed. New York: M. Dekker, 2001.

JANKOWSKY, I. P. et al. Madeiras brasileiras. Caxias do Sul: Spectrum, 1990. 172p.

LORENZI, H. Árvores brasileiras: manual de identificação e cultivo de plantas arbóreas nativas do Brasil. Nova Odessa: Plantarum, 1992. 352p.

Revista Árvore, Viçosa-MG, v.37, n.2, p.377-384, 2013 
PAULILO, M. T. S.; LAPA, F. S.; FALKENBERG, M. B. Effect of light intensity and growth substratum on plant development and production of secondary metabolites in Cordia curassavica (jacq.) Roem. \& Schult. Revista Árvore, v.34, n.3, p.417-423, 2010.

PASTORE, T. C. M. Estudos do efeito da radiação ultravioleta em madeiras por espectroscopias RAMAN (FTRAMAN), de refletância difusa no infravermelho (DRIFT) e no visível (CIE-L*a*b*). 2004. 131f. Tese (Doutorado em Química Analítica) - Universidade de Brasília, Brasília, 2004.
SILVA, J. O.; PASTORE, T. C. M. Fotodecomposição e proteção de madeiras tropicais. Floresta e Ambiente, v.21, n.2, p.7-13, 2004.

SILVA, J. O.; PASTORE, T. C. M.; PASTORE JÚNIOR, F. Resistência ao intemperismo artificial de cinco madeiras tropicais e de dois produtos de acabamento. Ciência Florestal, v.17, n.1, p.17-23, 2007.

SOUZA, H. N.; ARAUJO, T. G.; RIBEIRO, G. A. Avaliação da eficiência de um gel hidroretentor como retardante de fogo. Revista Árvore, v.36, n.3, p.471-477, 2012. 